\title{
Utilizing leadership to achieve high reliability in the delivery of perinatal care
}

\author{
This article was published in the following Dove Press journal: \\ Journal of Healthcare Leadership \\ 3I October 2012 \\ Number of times this article has been viewed
}

\section{Carmen Parrotta' \\ William Riley' \\ Les Meredith ${ }^{2}$}

'School of Public Health, University of Minnesota, Minneapolis, MN, ${ }^{2}$ Premier Insurance Management Services Inc, Charlotte, NC, USA
Correspondence: William Riley

Division of Health Policy and

Management, D 383 Mayo, 420 Delaware

Street SE, Minneapolis, MN 55455, USA

$\mathrm{Tel}+\mathrm{I} 6126250615$

Fax + I 612624 4408

Email riley00I@umn.edu
Abstract: Highly reliable care requires standardization of clinical practices and is a prerequisite for patient safety. However, standardization in complex hospital settings is extremely difficult to attain and health care leaders are challenged to create care delivery processes that ensure patient safety. Moreover, once high reliability is achieved in a hospital unit, it must be maintained to avoid process deterioration. This case study examines an intervention to implement care bundles (a collection of evidence-based practices) in four hospitals to achieve standardized care in perinatal units. The results show different patterns in the rate and magnitude of change within the hospitals to achieve high reliability. The study is part of a larger nationwide study of 16 hospitals to improve perinatal safety. Based on the findings, we discuss the role of leadership for implementing and sustaining high reliability to ensure freedom from unintended injury.

Keywords: care bundles, evidence-based practice, standardized care, process improvement

\section{Introduction}

Preventable perinatal harm occurs in hospitals with disturbing frequency and very few strategies have been developed to proactively reduce the system failures associated with perinatal incidents that cause preventable harm. High reliability is the study of safety successes rather than the failures in organizations. High reliability organizations (HROs) demonstrate the ability to operate technologically complex systems without error over long periods ${ }^{1}$ and can be distinguished by several common characteristics: (a) safety is the hallmark of organizational culture, (b) a team emphasis rather than individual performance with collegial rather than hierarchical interactions, (c) open and extensive communication is highly valued and rewarded, and (d) emergencies are rehearsed and the unexpected is practiced..$^{2-7}$ In high reliability organizations, managers expect to make errors and train their workforce to recognize and recover from them. ${ }^{8}$ In other words, HROs anticipate the worst and train to prevent these occurrences at all levels of the organization.

Achieving safety requires a health care system capable of preventing errors. Applying standardized protocols is commonplace in HROs, and its use has intensified in health care since being introduced over 15 years ago. ${ }^{9}$

It is well-documented that health care is not safe for patients ${ }^{10,11}$ and unintended perinatal harm has unique risk patterns. At least $1.5 \%$ of hospitalized obstetric patients experience an adverse event $\mathrm{t}^{12}$ and root cause analysis has associated communication failure with $72 \%$ of sentinel events in perinatal units. ${ }^{13}$ Despite tremendous individual commitment and the conscientious efforts of superbly trained professionals, high reliability is not a dominant feature of perinatal units in our health care system. ${ }^{14}$ 
Moreover, although obstetrics is extremely high risk, birth trauma is a low-frequency, high-severity problem that can result in catastrophic injury. The US ranks 17 th in the world in perinatal mortality rate - an outcome that according to the WHO is largely due to obstetric causes ${ }^{15}$ - and 29 th worldwide in infant mortality, near the bottom of the industrialized nations. ${ }^{16}$ Perinatal care is a high risk specialty, and no empirical evidence exists that demonstrates how implementing safe practices can reduce system failures that cause catastrophic patient harm.

To achieve high reliability, it is no longer sufficient for a health care organization to rely solely on the technical skills of providers. High reliability in health care is a function of technical skills, non-technical skills, and process design. ${ }^{17}$ Technical skills refer to the clinical abilities and knowledge of practitioners. These are the "hard skills" acquired through professional training in various disciplines such as medicine, nursing, and pharmacy and enhanced by experience and continuous education. Non-technical skills are the "soft skills" such as team communication, inter-professional performance, and leadership. These skills are less frequently taught as a professional skill and are acquired through onthe-job experience. Non-technical skills focus on interpersonal interaction, behavioral abilities, and interdisciplinary teamwork. ${ }^{18-20}$ Process design is the last component of high reliability. Technical skills and non-technical skills are limited by human factors including error, fatigue, mistakes, and perception. Therefore, processes must be designed to prevent and mitigate medical error caused by human factors as well as to standardize performance.

Becoming a high reliability organization also requires a culture of safety. A culture of safety is defined as the values patterns of behavior that determine the commitment to an organization's health and safety management. ${ }^{21}$ Figure 1 illustrates the relationship between high reliability organizations and their four components. Key leaders within an organization are responsible for fostering a culture of safety, modeling its practice, and aligning operational initiatives to demonstrate its importance.

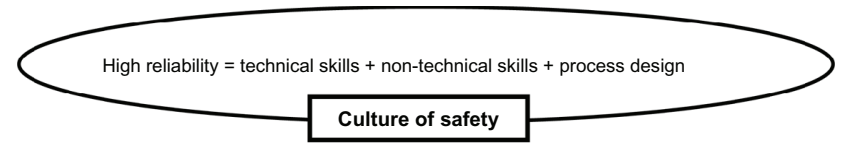

Figure I Conceptual model.

Note: Copyright (C) 2009, John Wiley and Sons. Reproduced with permission from Miller K, Riley W, Davis S. Identifying key nursing and team behaviours to achieve high reliability. J Nurs Manag. 2009; 17:247-255. ${ }^{17}$

\section{Care bundles}

A care bundle is a collection of evidence-based practices intended to effectively and safely care for patients undergoing particular treatments with inherent risks. ${ }^{22}$ Several evidencebased interventions shown to individually improve patient care are "bundled" together as a group to significantly improve patient care outcomes. The Institute for Healthcare Improvement (IHI) has developed three care bundles for perinatal care involving an elective bundle induction, labor augmentation, and non-surgical operative delivery. ${ }^{22}$

\section{Research methods}

This analysis is part of a larger study to improve perinatal safety in hospitals. The larger study is a quasi-experimental time-series analysis in 34 hospitals (16 intervention hospitals and 18 comparison group hospitals). The larger study has currently progressed over a 50-month period from March 2008 to May 2012.

\section{Intervention}

The intervention in the larger study is to implement three care bundles to achieve standardized delivery of care for three medical conditions in all perinatal units by all perinatal staff. The three perinatal care bundles are designed for elective inductions, labor augmentation, and vacuum-assisted deliveries. The elective induction bundle and augmentation bundle each consist of four items which must be carried out consistently, while the vacuum-assisted bundle consisted of five items. Table 1 shows the three care bundles and the interventions associated with each bundle.

Training and continuous education concerning approaches to adopt and implement care bundles was provided to clinical staff at each intervention hospital at the start of the project in 2008. Continuous technical support was also provided by the research team over the duration of this project. Each hospital tailored the methods of applying perinatal care bundles to best fit their organization and leadership styles. This allowed sites to maximize the likelihood of clinical adoption of perinatal bundles using an internal understanding of how to effectively motivate staff and incorporate new practices. For instance, one hospital developed physician bundle compliance scorecards for reporting performance about the induction, augmentation, and vacuum-delivery bundles. These reports were peerreviewed and created a level of transparency that promoted conformity with standardized practices. Another hospital required medical director pre-authorization when conducting an induction delivery prior to 39 weeks. Implementation methods like these varied across intervention hospitals and 
Table I Components of the perinatal care bundle

\begin{tabular}{lll}
\hline Elective induction & Augmentation & Vacuum \\
\hline - Gestational age $\geq 39$ weeks & - Documentation of estimated fetal weight & - Alternative labor strategies considered \\
- Normal fetal status (per NICHD tiers) & - Normal fetal status (per NICHD tiers) & - Patient prepared \\
prior to onset of oxytocin & - Pelvic exam prior to the onset of oxytocin & - High probability of success \\
- Pelvic exam prior to the onset & - Recognition and management & - Maximum application time and number of \\
of oxytocin & of tachysystole & pop-offs predetermined and documented \\
- Recognition and management & & - Cesarean and resuscitation teams available \\
of tachysystole & & at delivery \\
\hline
\end{tabular}

Abbreviation: NICHD, National Institute of Child Health and Human Development.

exemplified how leaders at each hospital utilized process design, within a culture of safety, to reduce practice variation for the perinatal care bundles studied in this project.

\section{Study setting}

From the 16 hospitals in the larger study, we identified four patterns of compliance, and we selected four hospitals that typify these patterns of change as they achieve process standardization. The four patterns are: (1) hospitals that significantly improved performance followed by a decline in performance, (2) hospitals that consistently perform well but deteriorate over time, (3) hospitals that significantly improve performance and maintain performance over time, and (4) hospitals that consistently perform well and maintain performance. Table 2 lists the four patterns of standardization and the characteristics (region of country, size of hospital, total annual deliveries) of each hospital selected to illustrate the pattern of change over time.

\section{Data collection}

Data were collected by auditing 20 randomly selected induction deliveries each month. The results of these bundle audits were de-identified and submitted by key personnel at each intervention hospital to a secure website. The research team then extracted this data for further analysis. To receive credit for compliance with a clinical practice bundle, all of the elements of the bundle must be met as evidenced in the monthly chart audits. Requiring "bundled" compliance with these best practices emphasizes the importance of standardized care to decrease variation and achieve high reliability.

\section{Data analysis}

The data are analyzed using run charts developed for Statistical Process Control (SPC). SPC is an analytic technique used to distinguish between routine (random) variation and unusual (unpredictable) variation to determine the performance of a process over time. ${ }^{23}$ Routine variation is known as common cause while unusual variation is known as special cause. SPC quantifies how a process is performing over time and uses a time-ordered sequence to determine what type of variation is present ${ }^{24}$ and whether the process is meeting desired performance targets. ${ }^{25}$ Run charts are a time-series representation of data used to track consistency of calculated statistical information generated from a variety of data sampling strategies. ${ }^{26}$ The usefulness of run charts lies in their ability to detect significant changes in a process. If special cause is detected in the process, then action can be directed at eliminating the variation. ${ }^{23,27}$

A run chart is based on the probability of observed sequences and Montgomery provides a more in-depth discussion of control chart interpretation. ${ }^{27}$ The centerline represents the median of the sample data, which reflects the central tendency of the sample data collected. We conducted four

Table 2 Characteristics of collaborative hospitals

\begin{tabular}{|c|c|c|c|c|c|}
\hline Hospital ID & $\begin{array}{l}\text { Bundle compliance } \\
\text { performance }\end{array}$ & $\begin{array}{l}\text { Number of similar } \\
\text { sites in collaborative }\end{array}$ & $\begin{array}{l}\text { Region } \\
\text { of country }\end{array}$ & $\begin{array}{l}\text { Number } \\
\text { of beds }\end{array}$ & $\begin{array}{l}\text { Annual } \\
\text { births }\end{array}$ \\
\hline A & $\begin{array}{l}\text { Significant improver } \\
\text { with deterioration }\end{array}$ & 2 & Midwest & 375 & 4132 \\
\hline B & $\begin{array}{l}\text { High performer with } \\
\text { deterioration }\end{array}$ & 2 & South & 261 & 912 \\
\hline C & $\begin{array}{l}\text { Significant improver } \\
\text { with high reliability }\end{array}$ & 2 & Midwest & 329 & 1823 \\
\hline D & $\begin{array}{l}\text { High performer with } \\
\text { high reliability }\end{array}$ & 4 & West & 250 & 2001 \\
\hline
\end{tabular}


Table 3 Statistical process control tests used in detecting the presence of special cause variation

\begin{tabular}{ll}
\hline Type of special cause & Definition \\
\hline $\begin{array}{l}\text { Number of runs } \\
\text { present }\end{array}$ & $\begin{array}{l}\text { A run is a collection of two or more } \\
\text { data points on one side of the center line; } \\
\text { control charts must contain between four } \\
\text { and eleven runs or special cause is present. } \\
\text { A run of more than eight consecutive } \\
\text { data points on one side of the center } \\
\text { line indicates special cause. } \\
\text { Seven or more consecutive ascending or } \\
\text { descending data points indicate special cause. }\end{array}$ \\
Zigzag pattern & $\begin{array}{l}\text { A repeating pattern of at least I } 4 \text { single } \\
\text { points alternating up and down across } \\
\text { the center line indicates special cause. }\end{array}$ \\
\hline
\end{tabular}

tests which indicate the presence of special cause variation. A process change is signified whenever a special cause is detected. Table 3 lists each of the four types of special cause and Lighter and Fair provide a detailed discussion about the statistical assumptions and requirements for determining special cause. $^{23}$

\section{Results}

We selected the elective induction bundle to analyze rates and magnitude of compliance. The figures below show a run chart analysis for induction bundle compliance rates of four separate hospitals over a 50-month period from March 2008 to May 2012. Each chart shows monthly compliance rates with all elements of the induction care bundle. The centerline is the median of the distribution. Special cause is indicated by the plot points circled on each chart.
Figure 2 shows a 78\% compliance rate for induction bundles in Hospital A during the 50-month period. The run chart indicates two special causes: (1) an 11-month period from March 2008 to February 2009 and (2) a 21-month period from February 2010 to October 2011. The run chart indicates that 25 months were required (March 2008 to March 2010) to achieve a process shift. However, the duration of the process shift was 19 months (March 2010 to October 2011). During the final 6 months, process deterioration occurred. Induction bundle compliance ranged from $0 \%$ to $100 \%$ for Hospital A.

The run chart in Figure 3 shows that Hospital B achieved a $98 \%$ induction bundle compliance rate over the 50-month period. Three special causes were detected, (1) a 12-month period (March 2008 through February 2009), (2) a 9-month period (December 2010 through August 2011), and (3) an 8 -month period (September 2011 through March 2012). The run chart indicates that 36 months were required for Hospital B to achieve a process shift. However, this process shift was followed by a significant decline as indicated by the third special cause. Induction bundle compliance for Hospital B ranged from $62 \%$ to $100 \%$.

Figure 4 shows the process performance for Hospital $\mathrm{C}$ during the same 50-month period. While the median compliance rate is $100 \%$, the mean is substantially less. The statistical assumptions required for a run chart disregard all observations that fall on the median. Therefore, only one occurrence of special cause was found: a 10-month period (March 2008 to December 2008). Induction bundle compliance ranged from 0 to $100 \%$ for Hospital C.

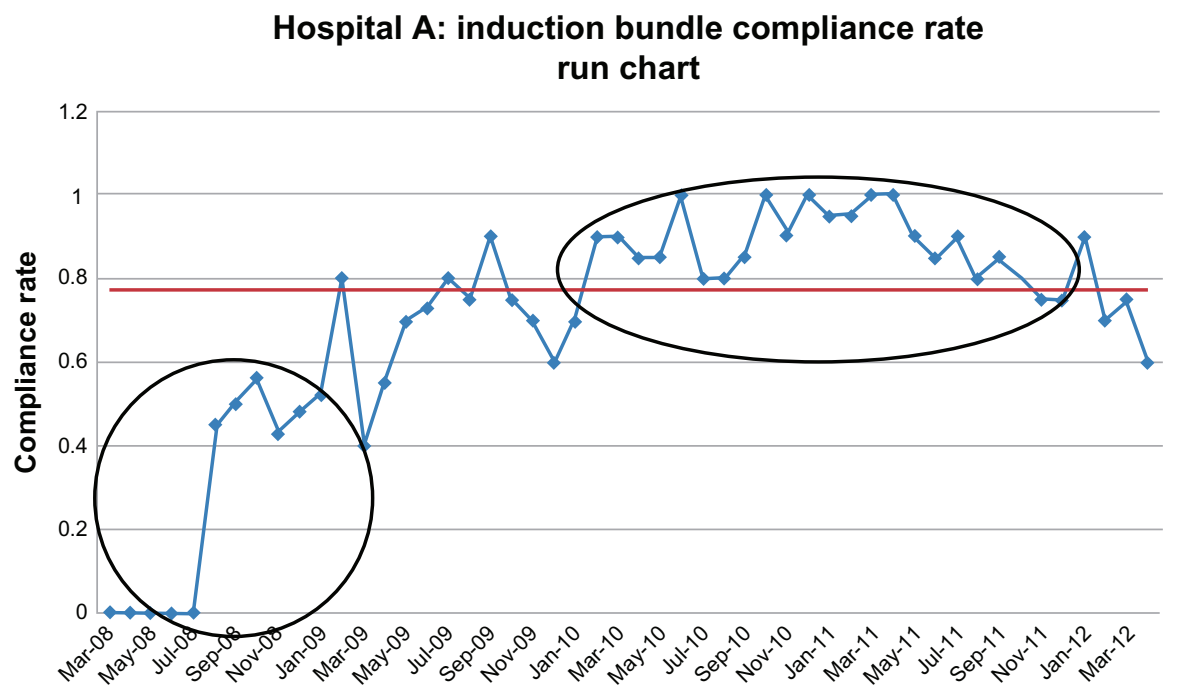

Figure 2 Induction bundle compliance rate. 


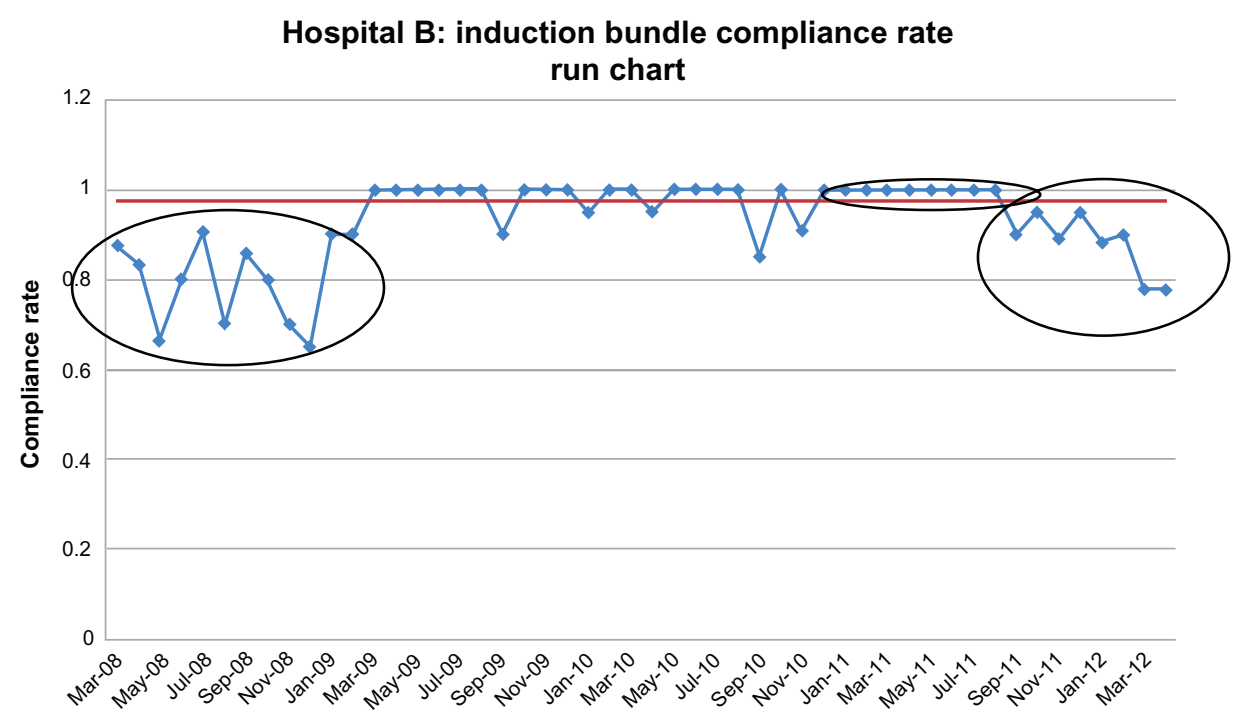

Figure 3 Induction bundle compliance rate.

The run chart analysis for Hospital D presented in Figure 5 shows a median induction bundle compliance rate of $92 \%$. Three instances of special cause were found in this process: (1) a 9-month period (March 2008 to November 2008), (2) a 13-month period (February 2009 to February 2010), and (3) a 13-month period (September 2010 to September 2011). Induction bundle compliance ranged from $61 \%$ to $100 \%$ for Hospital D.

\section{Discussion}

The four hospitals in this study have very different change patterns regarding bundle compliance over a 50-month intervention to implement standardized care for all deliveries involving elective induction. The behavior varied with respect to overall performance, the magnitude of change, and the rate of change. The summary in Table 4 shows that the median induction compliance rate for the four hospitals ranged from $78 \%$ to $100 \%$ while the induction bundle compliance ranged from $0 \%$ to $100 \%$. There does not appear to be a consistent relation between the median compliance rate of a hospital and its initial compliance level when the program intervention started. Moreover, despite the apparent lack of relationship between initial compliance rates and the median compliance, each of the four hospitals required approximately

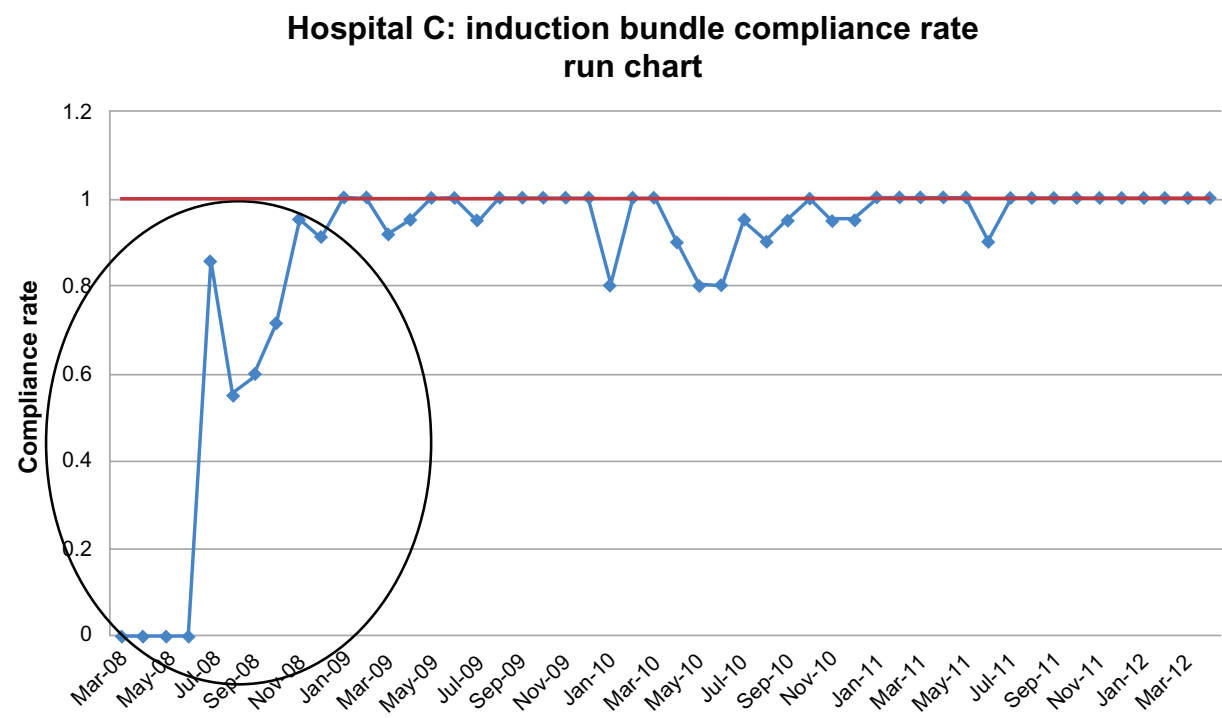

Figure 4 Induction bundle compliance rate. 


\section{Hospital D: induction bundle compliance rate run chart}

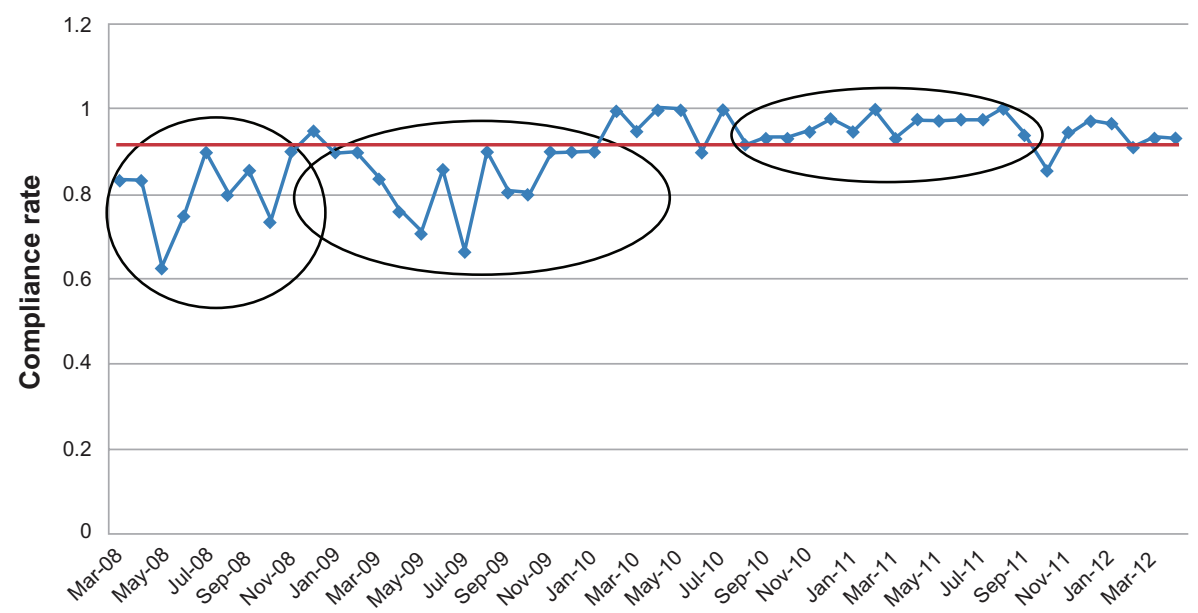

Figure $\mathbf{5}$ Induction bundle compliance rate.

1 year (10 months to 13 months) to achieve a process shift to its median performance.

The compliance rate for Hospital A reflects a common pattern seen in several hospitals in the larger study: very low compliance with the induction bundle early in the intervention, followed by a significant compliance increase. Although the compliance holds above the median for almost 2 years, it then drops. Hospital B is an example of a high-performing hospital at the start of the intervention, and therefore less improvement is attainable. This hospital experienced a drop in performance during the last 8 months. Hospital $\mathrm{C}$ is a significantly improved hospital that avoided any negative process trends and suggests that bundle compliance maintenance is feasible under the right conditions. Hospital D is a high-performing hospital at the start of the intervention with limited capacity to improve. While high reliability was not maintained at the end of the intervention period, there was no indication of a process shift.

\section{Process deterioration}

Organizations may experience process deterioration after an improvement initiative has been undertaken.

Table 4 Median induction compliance rates in sample hospital

\begin{tabular}{llll}
\hline Hospital & Median & Range & $\begin{array}{l}\text { Months to } \\
\text { achieve median }\end{array}$ \\
\hline A & $79 \%$ & $0 \%-100 \%$ & 12 months \\
B & $98 \%$ & $65 \%-100 \%$ & 13 months \\
C & $100 \%$ & $0 \%-100 \%$ & 11 months \\
D & $92 \%$ & $63 \%-100 \%$ & 10 months \\
\hline
\end{tabular}

In this case study, three of the four hospitals experienced a decline in induction bundle compliance once their own median performance was exceeded. This result is consistent with a recent study of 130 Veterans Affairs hospitals which found that after hospitals improved their performance in patient discharge planning, a majority experienced performance decline when the improvement program ended. ${ }^{28}$ This suggests that preemptive leadership is needed to avoid process deterioration once a process has been improved.

Entropy is a measure of the disorder present in a system. This concept might be applied to understanding organizational process performance. Like a piece of melting ice, care processes in health care can degrade or transform their states as environmental strains are introduced over time. Even intentionally designed processes at equilibrium can shift into a state of deterioration as conditions and time change. No matter how perfectly designed the process, without maintenance (or improvement), it will tend towards disorder and deteriorate. ${ }^{29}$ Leadership is vital to avoid such process deterioration. In learning organizations, a leader recognizes when the direction of the organization should be changed, and enables the organization to design processes to match the intended or desired outcomes. ${ }^{30}$

\section{Limitations}

The results of this study cannot be generalized. The four hospitals in this case study were purposefully selected and the 16 intervention hospitals in the larger study were not randomly selected. Next, the chart audits for the bundle compliance were done by staff at each hospital trained in the chart audit methodology developed for this initiative. It is possible 
that there was variation in the interpretation of charts from the various auditors in the 16 hospitals. Finally, we did not study leadership as a part of this project. The impact of leadership on both the improvement and decline of induction bundle compliance is inferred in our discussion.

\section{Conclusion}

The introduction of standardized care bundles to improve patient safety is a promising development to promote patient safety. The findings in this case study indicate that the rate and magnitude of change to implement elective induction bundles are not consistent and vary widely. Leaders have the opportunity to leverage performance improvement initiatives in order to create meaningful and lasting change in patient safety by ensuring that standardized care processes are implemented and monitored so as to avoid process deterioration once improvement targets have been met.

\section{Disclosure}

The authors have no conflicts of interest to declare.

\section{References}

1. Roberts KH. Some characteristics of one type of high reliability organizations. Organization Science. 1990;1(2):160-177.

2. Weick KE, Sutcliffe KM. Managing the Unexpected: Assuring High Performance in an Age of Complexity. San Francisco, CA: Jossey-Bass; 2001.

3. Roberts KH, Stout SK, Halpern JJ. Decision dynamics in two high reliability military organizations. Management Science. 1994;40(5):614-624.

4. Klein RL, Bigley JA, Roberts KH. Organizational culture in high reliability organizations: an extension. Human Relations. 1995;48(7):771-793.

5. Roberts KH. Managing hazardous organizations. California Management Review. 1990;32:101-113.

6. Weick KE, Sutcliffe KM, Obstfeld D. Organizing for high reliability: Processes of collective mindfulness. Research in Organizational Behavior. 1999;21:81-123.

7. Weick KE. The vulnerable system: an analysis of the Tenerife air disaster. Journal of Management. 1990;16(3):571-593.

8. Reason J. Managing the Risks of Organizational Accidents. Cornwall, UK: Ashgate Publishing; 1997.

9. Nolan T, Resar R, Haraden C, Griffin FA. Improving the Reliability of Health Care. IHI Innovation Series white paper. Boston, MA: Institute for Healthcare Improvement; 2004.

10. Cherouny PH, Federico FA, Haraden C, Leavitt S, Resar R. Idealized design of perinatal care. Cambridge, MA: Institute for Healthcare Improvement; 2005.

11. McGlynn EA, Asch SM, Adams J, et al. The quality of health care delivered to adults in the United States. $N$ Engl J Med. 2003; 348(26):2635-2645.

Journal of Healthcare Leadership

\section{Publish your work in this journal}

The Journal of Healthcare Leadership is an international, peer-reviewed, open access journal focusing on leadership for the health profession. The journal is committed to the rapid publication of research focusing on but not limited to: Healthcare policy and law; Theoretical and practical aspects healthcare delivery; Interactions between healthcare and society and evidence-based practices;
12. Leape LL, Brennan TA, Laird NM, Lawthers AG, Localio AR, Barnes BA Incidence of adverse events and negligence in hospitalized patients. Results of the Harvard Medical Practice Study II. $N$ Engl J Med. 1991;324:377-384.

13. The Joint Commission. Sentinel Event Alert, Issue 30: Preventing infant death and injury during delivery. Available at: http://www. jointcommission.org/sentinel_event_alert_issue_30_preventing_infant_ death_and_injury_during_delivery/. Accessed September 25, 2009.

14. Miller K, Riley W, Davis S. Identifying key nursing and team behaviours to achieve high reliability. J Nurs Manag. 2009;17:247-255.

15. World Health Organization. Neonatal and Perinatal Mortality: Country, Regional and Global Estimates. Geneva; WHO; 2006. Available at: http://whqlibdoc.who.int/publications/2006/9241563206_eng.pdf. Accessed on January 13, 2010.

16. Mathews TJ, MacDorman MF. Infant mortality statistics from the 2005 period linked birth/infant death data set. Natl Vital Stat Rep. 2008;57(2):1-32.

17. Riley W, Davis SE, Miller KK, McCullough M. A model for developing high-reliability teams. J Nurs Manag. 2010;18(5):556-563.

18. Riley W, Hansen H, Gurses A, Davis S, Miller K, Priester R. The nature, characteristics and patterns of perinatal critical events teams. In: Henriksen L, Battles JB, Keyes MA, Grady ML, editors. Advances in Patient Safety: New Directions and Administrative Approaches. Rockville, MD: Agency for Healthcare Research and Quality; 2008: 131-144.

19. Singer SJ, Gaba DM, Geppert JJ, Sinaiko AD, Howard SK, Park KC. The culture of safety: results of an organization-wide survey in 15 California hospitals. Qual Saf Health Care. 2003;12(2):112-118.

20. Doyle JL, Kenny TH, von Gruenigen VE, Butz AM, Burkett AM. Implementing an induction schedule procedure and consent form to improve quality of care. Journal of J Obstet Gynecol Neonatal Nurs. 2012;41(4):462-473.

21. Burke CS, Salas E, Wilson-Donnelly K, Priest H. How to turn a team of experts into an expert medical team: guidance from the aviation and military communities. Qual Saf Health Care. 2004;13 Suppl 1: i96-i104.

22. The Joint Commission. Raising the bar with bundles: Treating patients with an all-or-nothing approach. Joint Commission Perspectives on Patient Safety. 2006;6(4):5-6.

23. Lighter DE, Fair DC. Quality Management in Health Care: Principles and Methods. 2nd ed. Sudbury, MA: Jones and Bartlett Publishers; 2004.

24. Carey RG. Improving Healthcare With Control Charts: Basic and Advanced SPC Methods and Case Studies. Milwaukee, WI: ASQ Quality Press; 2003.

25. Wheeler DJ. Understanding Variation: The Key to Managing Chaos. 2nd ed. Knoxville, TN: SPC Press; 2000.

26. Hart MK, Hart RF. Statistical Process Control for Health Care. Duxbury, MA: Thomson Learning; 2002.

27. Montgomery DC. Introduction to Statistical Quality Control. 5th ed. Hoboken, NJ: John Wiley and Sons Inc; 2005.

28. Glasgow JM, Davies ML, Kaboli PJ. Findings from a national improvement collaborative: are improvements sustained? Br Med J Qual Saf. 2012;21(8):663-669. doi: 10.1136/bmjqs-2011-000243.

29. Berwick DM. Escape Fire: Designs for the Future of Health Care. San Francisco, CA: Jossey-Bass; 2004.

30. Joiner B. Fourth Generation Management: The New Business Consciousness. New York, NY: McGraw-Hill International Book Co; 1994.

\section{Dovepress}

Interdisciplinary decision-making; Philosophical and ethical issues; Hazard management; Research and opinion for health leadership; Leadership assessment. The manuscript management system is completely online and includes a very quick and fair peer-review system. Visit http://www.dovepress.com/ testimonials.php to read real quotes from published authors. 